\title{
New relations among associated Legendre functions and spherical harmonics
}

\author{
S. J. Claessens \\ Western Australian Centre for Geodesy, Curtin University of Technology, GPO Box U1987, 6845, Perth, WA, Australia \\ e-mail: claesses@vesta. curtin.edu.au; Tel.: +61 89266 2218; Fax: +61 892662703
}

Received: 30 September 2004 / Accepted: 4 July 2005 / Published online: 4 August 2005

\begin{abstract}
Several new relations among associated Legendre functions (ALFs) are derived, most of which relate a product of an ALF with trigonometric functions to a weighted summation over ALFs, where the weights only depend on the degree and order of the ALF. These relations are, for example, useful in applications such as the computation of geopotential coefficients and computation of ellipsoidal corrections in geoid modelling. The main relations are pre-

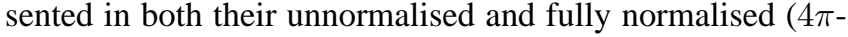
normalised) form. Several approaches to compute the weights involved are discussed, and it is shown that the relations can also be applied in the case of first- and second-order derivatives of ALFs, which may be of use in analysis of satellite gradiometry data. Finally, the derived relations are combined to provide new identities among ALFs, which contain no dependency on the colatitudinal coordinate other than that in the ALFs themselves.
\end{abstract}

Key words. associated Legendre functions - spherical harmonics

\section{Introduction}

Surface spherical harmonic functions, often simply called spherical harmonics, form the most commonly used base functions for representation of the Earth's gravity field (e.g. Lemoine et al. 1998; Reigber et al. 2002) and are also used in many other branches of physics, such as nuclear and atomic physics (e.g. Blatt and Weisskopf 1962), electromagnetics (e.g. Rothwell and Cloud 2001) and astronomy (e.g. Percival et al. 2004). Their mathematical properties, in particular those of the underlying associated Legendre functions (ALFs), have therefore been studied in great detail by various authors (e.g. Hobson 1931; MacRobert 1967) and this has led to many relations among them. A subset of these relations equate the product of an ALF and a function that depends on the same variable as the ALF, in geodesy often the latitude $\varphi$ or co-latitude $\theta$, to a summation of weighted ALFs. The weights of the ALFs in the summation only depend on the degree and order of the ALFs in question. These relations are particularly useful, mainly because ALFs are often contained within an integral over the angle that the ALFs are dependent on (e.g. Gerstl 1980). Using these type of equations, any other dependence on this angle inside the integral can be completely removed, which is the key to their use in many applications.

One particular case is the so-called product-sum formula of ALFs (e.g. Giacaglia 1980; Hwang 1995), which involves the multiplication of two ALFs. Other examples include the product of an ALF with the trigonometric functions sine, cosine and cotangent. These follow directly from recursive relations among ALFs that are often utilised to compute them and are therefore ubiquitous in literature (e.g. Hobson 1931; MacRobert 1967; Abramowitz and Stegun 1972). Applying these formulae twice results in an expression for the case of squares of trigonometric functions, as is for example performed in Moritz (1980, Eq. 39-76) to compute ellipsoidal corrections to gravity anomalies.

In this paper, general relations for arbitrary powers of trigonometric functions are presented, for which the weight functions can be computed recursively. These relations can improve the accuracy of ellipsoidal corrections in geoid computation by simple extensions of the work presented in Martinec (1998), Heck and Seitz (2003) and Sjöberg (2003, 2004). They are even more vital in the computation of geopotential coefficients, extending on the work by Cruz (1986), Petrovskaya et al. (2001) and Sjöberg (2003), as has been shown in Claessens and Featherstone (2005). The derived relations are also extended to first- and second-order derivatives of ALFs, which could prove useful in for example analysis of satellite gradiometry data. Finally, several new identities among ALFs are obtained.

\section{Basic relations}

The surface spherical harmonic functions $Y_{n m}(\theta, \lambda)$ of degree $n$ and order $m$ are defined as

$Y_{n m}(\theta, \lambda)=P_{n|m|}(\cos \theta)\left\{\begin{array}{l}\cos m \lambda \text { for } m \geq 0 \\ \sin |m| \lambda \text { for } m<0\end{array}\right.$

where $\theta$ and $\lambda$ are the co-latitude and longitude respectively, and $P_{n m}$ are the ALFs, which obey the characteristic dif- 
ferential equation based on Laplace's equation (e.g. Hobson 1931).

Several recurrence relations among ALFs are known (e.g. MacRobert 1967; Abramowitz and Stegun 1972)

$$
\begin{array}{r}
P_{n m}=(2 n-1) \sin \theta P_{n-1, m-1}+P_{n-2, m} \\
P_{n m}=\frac{2 n-1}{n-m} \cos \theta P_{n-1, m}-\frac{n+m-1}{n-m} P_{n-2, m}, \\
m \leq n-1
\end{array}
$$

$$
\begin{aligned}
P_{n m} & =2(m-1) \cot \theta P_{n, m-1}-(n-m+2) \\
& \times(n+m-1) P_{n, m-2}, \quad m \geq 2
\end{aligned}
$$

These equations and all equations below at least hold for the ranges $n \geq 2$ and $0 \leq m \leq n$, unless otherwise stated. ALFs with $m>n$ are defined as equal to zero.

ALFs are often fully normalised, making the average square value of the harmonics over the sphere equal to 1 , by applying a scale factor based on the degree $n$ and order $m$ of the function

$\bar{P}_{n m}=P_{n m} \begin{cases}\sqrt{2 n+1} & \text { for } m=0 \\ \sqrt{2(2 n+1) \frac{(n-m) !}{(n+m) !}} & \text { for } m \neq 0\end{cases}$

and introducing the fully normalised ALFs into Eq. (1) yields fully normalised spherical harmonics $\bar{Y}_{n m}$. Algorithms to compute fully normalised ALFs, avoiding numerical instabilities, are provided by Koop and Stelpstra (1989) and Holmes and Featherstone (2002).

Equations (2) to (4) can be transformed in such a way that the product of a trigonometric function and an ALF is expressed as a weighted sum over two other ALFs, where the weights are independent of angle $\theta$. For Eq. (3), for example, this gives Eq. (39-45) in Moritz (1980)

$\cos \theta P_{n m}=F_{n m}^{-1} P_{n-1, m}+F_{n m}^{1} P_{n+1, m}$

where

$F_{n m}^{-1}=\frac{n+m}{2 n+1}$ and $F_{n m}^{1}=\frac{n-m+1}{2 n+1}$

Eq. (6) can be transformed into a fully normalised form using Eq. (5), yielding

$\cos \theta \bar{P}_{n m}=\bar{F}_{n m}^{-1} \bar{P}_{n-1, m}+\bar{F}_{n m}^{1} \bar{P}_{n+1, m}$

where

$\bar{F}_{n m}^{-1}=\sqrt{\frac{(n+m)(n-m)}{(2 n-1)(2 n+1)}}$

$\bar{F}_{n m}^{1}=\sqrt{\frac{(n-m+1)(n+m+1)}{(2 n+1)(2 n+3)}}$

The same approach can be followed for Eqs. (2) and (4), and the resulting formulae can be found in Table 1.

From Eq. (1), it can be seen that Eqs. (6) and (8) are also valid when $P_{n m}$ and $\bar{P}_{n m}$ are replaced by the spherical harmonics $Y_{n m}$ and $\bar{Y}_{n m}$ respectively, since the ALFs are of equal order $m$.

\section{New relations}

As noted in, for example, Moritz (1980), Eq. (6) can be applied twice to obtain a relation when the cosine is squared. This results in

$\cos ^{2} \theta P_{n m}=F_{n m}^{-2} P_{n-2, m}+F_{n m}^{0} P_{n m}+F_{n m}^{2} P_{n+2, m}$

where

$F_{n m}^{-2}=\frac{(n+m)(n+m-1)}{(2 n-1)(2 n+1)}$

$F_{n m}^{0}=\frac{2 n^{2}-2 m^{2}+2 n-1}{(2 n-1)(2 n+3)}$

$F_{n m}^{2}=\frac{(n-m+1)(n-m+2)}{(2 n+1)(2 n+3)}$

Relations for fully normalised ALFs, as well as for the cases involving the sine and the cotangent, can also easily be derived in the same way, and are shown in Table 2. The use of some trigonometric identities can yield even more relations. For example, since the sum of the squares of the sine and cosine of the same angle are equal to 1, it follows from Eq. (11) that

$\sin ^{2} \theta P_{n m}=K_{n m}^{-2} P_{n-2, m}+K_{n m}^{0} P_{n m}+K_{n m}^{2} P_{n+2, m}$

where

$K_{n m}^{-2}=-F_{n m}^{-2}, \quad K_{n m}^{0}=1-F_{n m}^{0}, \quad K_{n m}^{2}=-F_{n m}^{2}$

In general, for any power of the cosine term, its product with an ALF (or similarly with a spherical harmonic) can be expressed as a weighted summation over ALFs (or spherical harmonics) of equal order

$\cos ^{j} \theta P_{n m}=\sum_{i=-j, 2}^{j} F_{n m}^{i j} P_{n+i, m}, \quad j \in \mathbb{N}$

where the coefficients $F_{n m}^{i j}$ depend on degree $n$ and order $m$ solely and $\mathbb{N}$ is the set of natural numbers. The summation runs from $-j$ to $j$ in steps of 2 . Along the same line of reasoning, similar equations can be derived for the cases involving a power of the sine and the cotangent.

Now the question arises how to compute the coefficients $F_{n m}^{i j}$ in Eq. (17). A recursive relation can be found by investigation of the scheme shown in Fig. 1. The coefficients of index $j$ can be obtained by applying Eq. (6) to the previous level of index $j-1$. For example, coefficient $F_{n m}^{-2,2}$ is multiplied by ALF $P_{n-2, m}$ and when this is multiplied with another cosine of $\theta$, this term becomes, with Eq. (6)

$F_{n m}^{-2,2} F_{n-2, m}^{-1,1} P_{n-3, m}+F_{n m}^{-2,2} F_{n-2, m}^{1,1} P_{n-1, m}$

Thus, $F_{n m}^{-3,3}=F_{n m}^{-2,2} F_{n-2, m}^{-1,1}$, and $F_{n m}^{-1,3}$ is the sum of $F_{n m}^{-2,2} F_{n-2, m}^{1,1}$ plus an extra term from $F_{n m}^{0,2}$ (see Fig. 1). In general, the arrows pointing left in Fig. 1 indicate a multiplication of the coefficient with $F_{n+i-1, m}^{-1,1}$ and the arrows 


\begin{tabular}{lll}
\hline $\sin \theta P_{n m}=E_{n}^{-1} P_{n-1, m+1}+E_{n m}^{1} P_{n+1, m+1}$ & $E_{n}^{-1}=-\frac{1}{2 n+1}$ & $\bar{E}_{n m}^{-1}=-\sqrt{\frac{(n-m)(n-m-1)}{\left(\delta_{0, m}+1\right)(2 n-1)(2 n+1)}}$ \\
$\cos \theta P_{n m}=F_{n m}^{-1} P_{n-1, m}+F_{n m}^{1} P_{n+1, m}$ & $E_{n}^{1}=\frac{1}{2 n+1}$ & $\bar{E}_{n m}^{1}=\sqrt{\frac{(n+m+1)(n+m+2)}{\left(\delta_{0, m}+1\right)(2 n+1)(2 n+3)}}$ \\
$\cot \theta P_{n m}^{-1}=\frac{n+m}{2 n+1}$ & $\bar{F}_{n m}^{-1}=\sqrt{\frac{(n+m)(n-m)}{(2 n-1)(2 n+1)}}$ \\
& $F_{n m}^{1}=\frac{n-m+1}{2 n+1}$ & $\bar{F}_{n m}^{1}=\sqrt{\frac{(n-m+1)(n+m+1)}{(2 n+1)(2 n+3)}}$ \\
\hline
\end{tabular}

Table 1. Relations involving the product of a trigonometric function and an ALF expressed as a weighted summation over two other ALFs, where the weights are given for the unnormalised case (middle column) and the fully normalised case (right column), where $\delta_{i j}$ is the Kronecker delta

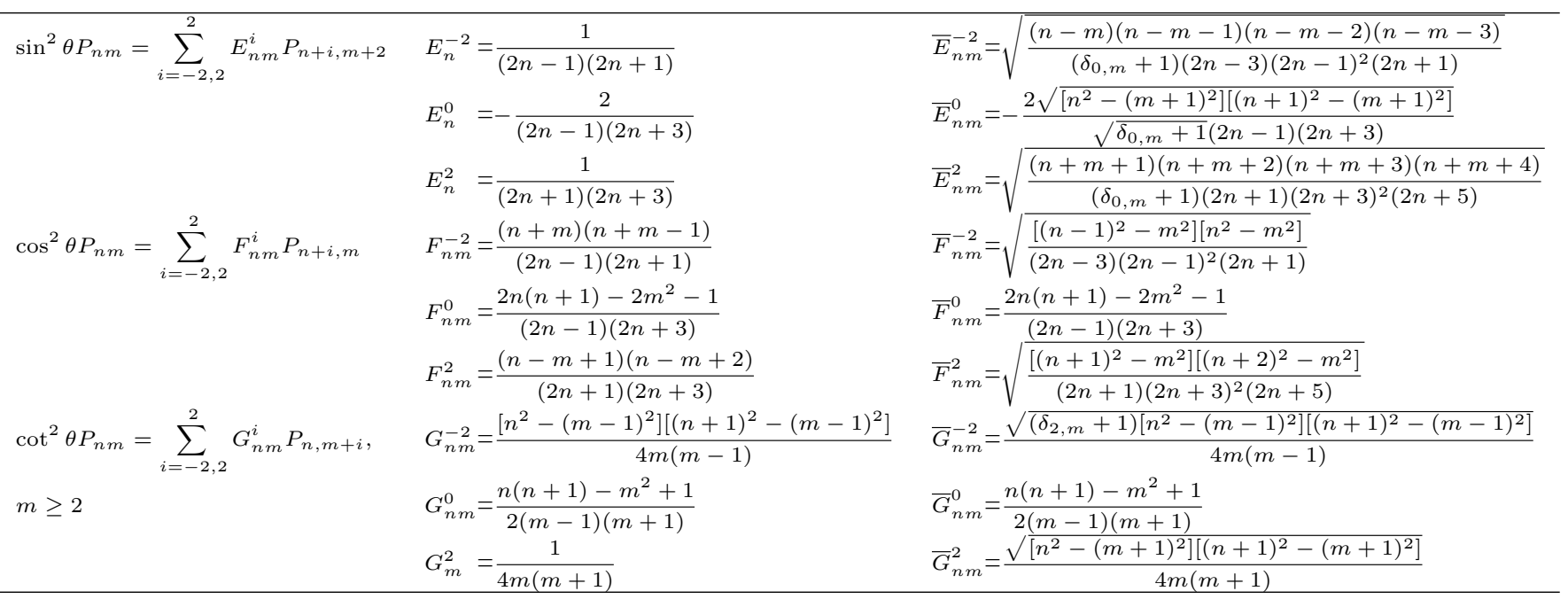

Table 2. Relations involving the product of the square of a trigonometric function and an ALF expressed as a weighted summation over three other ALFs, where the weights are given for the unnormalised case (middle column) and the fully normalised case (right column)

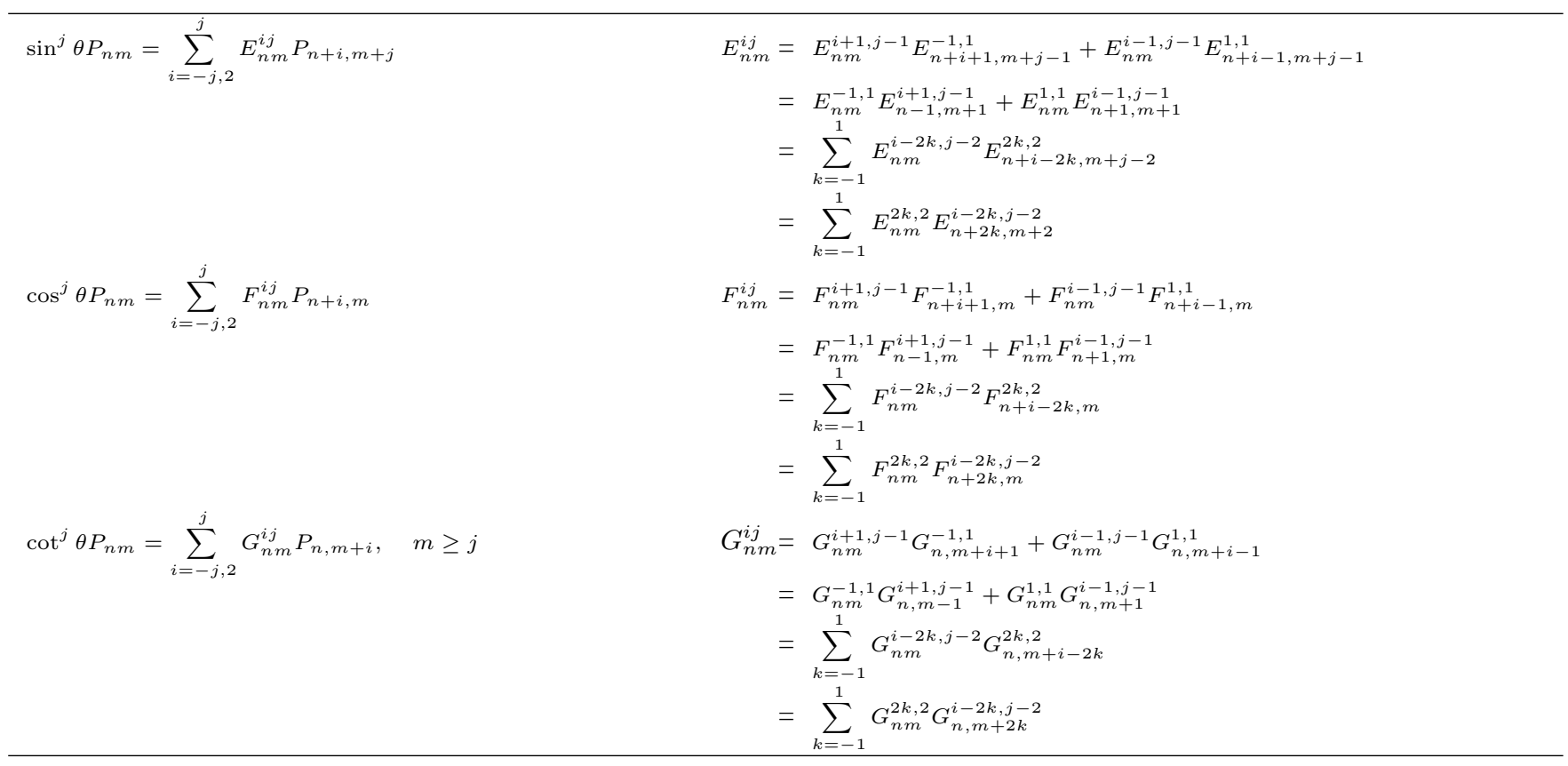

Table 3. Relations involving the product of an arbitrary power of a trigonometric function and an ALF expressed as a weighted summation over two or more other ALFs. The weights can be computed recursively from the initial values given in Table 1 or 2 using the relations given here, which are all valid for both unnormalised and fully normalised ALFs 


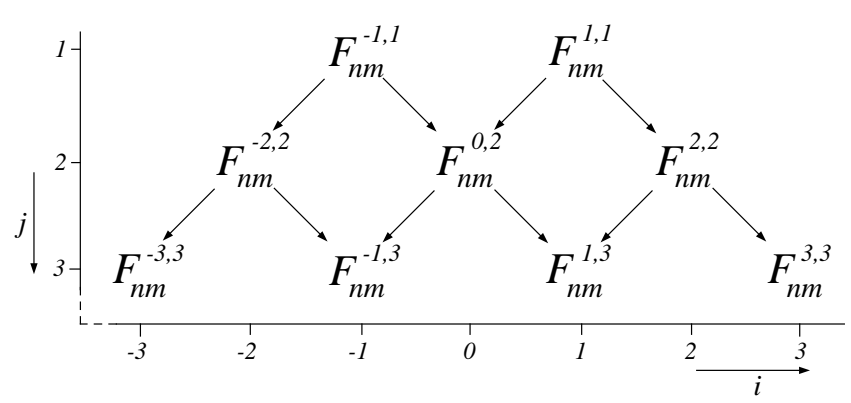

Figure 1. Recursive computation of coefficients $F_{n m}^{i j}$

pointing right indicate a multiplication of the coefficient with $F_{n+i-1, m}^{1,1}$ This leads to the following recursive relation

$F_{n m}^{i j}=F_{n m}^{i-1, j-1} F_{n+i-1, m}^{1,1}+F_{n m}^{i+1, j-1} F_{n+i+1, m}^{-1,1}$

where $F_{n m}^{i j}$ are set to zero for $|i|>j$ or $n<m$. This recursive relation and all relations below hold for the ranges $j \geq 2$ and $-j \leq i \leq j$, unless otherwise stated, where it should be noted that $i$ and $j$ are either both even or both odd. Eq. (19) can also be found in a purely algebraic way, as follows

$$
\begin{aligned}
\cos ^{j} \theta P_{n m} & =\cos \theta \cos ^{j-1} \theta P_{n m} \\
& =\sum_{p=-j+1,2}^{j-1} F_{n m}^{p, j-1} \cos \theta P_{n+p, m} \\
& =\sum_{p=-j+1,2}^{j-1} F_{n m}^{p, j-1} \sum_{q=-1,2}^{1} F_{n+p, m}^{q, 1} P_{n+p+q, m}
\end{aligned}
$$

Substituting $p$ by $i-q$, this results in

$\cos ^{j} \theta P_{n m}=\sum_{i=-j, 2}^{j} \sum_{q=-1,2}^{1} F_{n m}^{i-q, j-1} F_{n+i-q, m}^{q, 1} P_{n+i, m}$

and upon comparison with Eq. (17), a relation for the coefficient $F_{n m}^{i j}$ emerges as

$F_{n m}^{i j}=F_{n m}^{i+1, j-1} F_{n+i+1, m}^{-1,1}+F_{n m}^{i-1, j-1} F_{n+i-1, m}^{1,1}$

which is exactly the same as Eq. (19). However, if the terms $\cos \theta$ and $\cos ^{j-1} \theta$ in Eq. (20) are reversed, another recursive relation appears as

$$
\begin{aligned}
\cos ^{j} \theta P_{n m} & =\cos ^{j-1} \theta \cos \theta P_{n m} \\
& =\sum_{q=-1,2}^{1} F_{n m}^{q, 1} \sum_{p=-j+1,2}^{j-1} F_{n+q, m}^{p, j-1} P_{n+p+q, m} \\
& =\sum_{i=-j, 2}^{j} \sum_{q=-1,2}^{1} F_{n m}^{q, 1} F_{n+q, m}^{i-q, j-1} P_{n+i, m}
\end{aligned}
$$

Thus

$F_{n m}^{i j}=F_{n m}^{-1,1} F_{n-1, m}^{i+1, j-1}+F_{n m}^{1,1} F_{n+1, m}^{i-1, j-1}$

Equations (22) and (24) can both be used to compute any coefficient from the initial values in Eq. (7). Equation (22) has

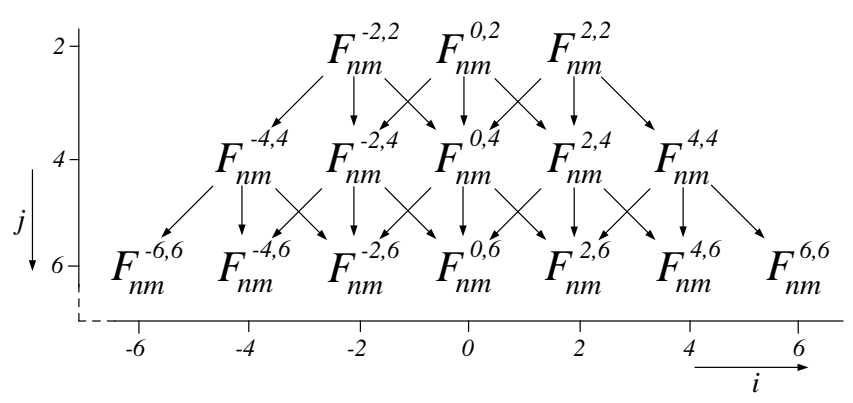

Figure 2. Recursive computation of coefficients $F_{n m}^{i j}$ for even $j$

the advantage that the coefficient of one solitary pair of degree $n$ and order $m$ can be evaluated very quickly since only the initial values $(j=1)$ need to be known for other degrees. These initial values, $F_{n+i+1, m}^{-1,1}$ and $F_{n+i-1, m}^{1,1}$, can be directly computed from the equations given in Table 1 . Thus, only a recursion over $i$ and $j$ is needed. Equation (24), on the other hand, requires knowledge of coefficients of degrees $n-1$ and $n+1$ with index $j-1\left(F_{n-1, m}^{i+1, j-1}\right.$ and $\left.F_{n+1, m}^{i-1, j-1}\right)$. These coefficients in their turn depend upon coefficients of degree $n-2$, $n$, and $n+2$, with index $j-2$. A recursive scheme based on this equation should therefore compute many coefficients of various degrees with indices up to $j-1$, before the coefficients of higher index $j$ can be computed. However, if all coefficients from degree and order zero onwards are desired, Eq. (24) can provide a slightly more efficient recursion than Eq. (22), because the initial values don't need to be stored or computed as often.

If one is only interested in coefficients of even $j$, as is for example the case in the computation of geopotential coefficients (Claessens and Featherstone 2005), other recursive relations can be found by taking the case $j=2$ as initial values. A recursive scheme is shown in Fig. 2 and the mathematical relation can easily be formulated

$F_{n m}^{i j}=\sum_{k=-1}^{1} F_{n m}^{i-2 k, j-2} F_{n+i-2 k, m}^{2 k, 2}$

Also in this case, an alternative expression can be found along similar lines as Eqs. (23) to (24), which is

$F_{n m}^{i j}=\sum_{k=-1}^{1} F_{n m}^{2 k, 2} F_{n+2 k, m}^{i-2 k, j-2}$

Recursive computation based on Eqs. (25) and (26) instead of Eqs. (22) and (24) is more efficient, since it avoids having to compute the coefficients of odd index $j$ in the process.

Thus, the computation of the coefficients $F_{n m}^{i j}$ is very flexible and can be performed in many ways. Moreover, numerical tests based on Eqs. (25) and (26) have shown that the computation of the coefficients can be done with high accuracy for even very high degree and order (up to $n=m=$ 2700) using either equation. The relative error in Eq. (17) was computed and this was found to be largely independent of degree $n$ and order $m$, although a very slight degradation of accuracy can be observed for orders $m$ approaching degree $n$. A more serious effect is the loss of accuracy for coefficients of higher index $j$. The absolute relative errors for $j$ up to 32 


\begin{tabular}{cccccccc}
$j$ & $E_{n m}^{i j}$ (analytic) & $E_{n m}^{i j}$ (recursive) & $\bar{E}_{n m}^{i j}$ & $F_{n m}^{i j}$ & $\bar{F}_{n m}^{i j}$ & $G_{n m}^{i j}$ & $\bar{G}_{n m}^{i j}$ \\
\hline 2 & $3.5 \cdot 10^{-14}$ & $3.5 \cdot 10^{-14}$ & $3.5 \cdot 10^{-14}$ & $6.8 \cdot 10^{-16}$ & $1.0 \cdot 10^{-15}$ & $5.0 \cdot 10^{-14}$ & $6.7 \cdot 10^{-14}$ \\
4 & $3.9 \cdot 10^{-14}$ & $3.8 \cdot 10^{-14}$ & $6.1 \cdot 10^{-14}$ & $2.1 \cdot 10^{-15}$ & $1.3 \cdot 10^{-15}$ & $8.3 \cdot 10^{-13}$ & $1.1 \cdot 10^{-12}$ \\
8 & $3.0 \cdot 10^{-14}$ & $3.0 \cdot 10^{-14}$ & $4.7 \cdot 10^{-14}$ & $5.7 \cdot 10^{-15}$ & $4.5 \cdot 10^{-15}$ & $8.5 \cdot 10^{-10}$ & $1.4 \cdot 10^{-09}$ \\
16 & $4.9 \cdot 10^{-13}$ & $4.9 \cdot 10^{-13}$ & $1.1 \cdot 10^{-13}$ & $3.7 \cdot 10^{-14}$ & $3.0 \cdot 10^{-14}$ & $9.9 \cdot 10^{-03}$ & $1.6 \cdot 10^{-02}$ \\
32 & $1.7 \cdot 10^{-04}$ & $1.7 \cdot 10^{-04}$ & $1.5 \cdot 10^{-11}$ & $3.6 \cdot 10^{-12}$ & $3.4 \cdot 10^{-12}$ & $2.0 \cdot 10^{-01}$ & $2.2 \cdot 10^{-01}$
\end{tabular}

Table 4. Average absolute relative errors per index $j$ in the equations in the left-hand column of Table 3 for the unnormalised and fully normalised case for degree $n$ and order $m$ up to 360 with $\theta=45^{\circ}$, where the coefficients were computed in 64 -bits double precision $(\sim 16$ digits) using the fourth, eight and twelfth equation in the right-hand column of Table 3 with the initial values in Table 2 , as well as using the analytic formula for $E_{n m}^{i j}$ in Eq. (34)

are shown in Table 4 for both the unnormalised and the fully normalised case.

For even $j$, also for the case of $\sin ^{j} \theta$, an expression can be found

$\sin ^{j} \theta P_{n m}=\sum_{i=-j, 2}^{j} K_{n m}^{i j} P_{n+i, m}, \quad j \in \mathbb{E}$

where $\mathbb{E}$ is the set of even natural numbers and the coefficients $K_{n m}^{i j}$ can be computed with the recursive relations in Eqs. (25) and (26), where $F$ is replaced by $K$. The initial values in this case are

$K_{n m}^{-2,2}=-F_{n m}^{-2,2}, \quad K_{n m}^{0,2}=1-F_{n m}^{0,2}, \quad K_{n m}^{2,2}=-F_{n m}^{2,2}$

which simply follows from the fact that $\sin ^{2} \theta+\cos ^{2} \theta=1$.

In the case of fully normalised ALFs or spherical harmonics, the recursive relations for the computation of the coefficients are exactly the same. More importantly, the initial values in Eqs. (9) and (10) are different, and because of this, the fully normalised coefficients $\bar{F}_{n m}^{i j}$ possess an interesting property that can facilitate their computation: the two initial values are connected by a simple equation

$\bar{F}_{n m}^{1,1}=\bar{F}_{n+1, m}^{-1,1}$

This relation can be generalised into

$\bar{F}_{n m}^{i j}=\bar{F}_{n+i, m}^{-i, j}$

A proof of Eq. (30) based on mathematical induction can be found in Appendix A. Eq. (30) can reduce the necessary computation time approximately by a factor 2 , since only coefficients with $i \geq 0$ need to be computed. The coefficients of negative index $i$ then follow directly from Eq. (30). Moreover, using Eq. (30), Eqs. (22) and (25) can be formulated in a slightly more convenient way

$\bar{F}_{n m}^{i j}=\bar{F}_{n m}^{i+1, j-1} \bar{F}_{n+i, m}^{1,1}+\bar{F}_{n m}^{i-1, j-1} \bar{F}_{n+i, m}^{-1,1}$

$\bar{F}_{n m}^{i j}=\sum_{k=-1}^{1} \bar{F}_{n m}^{i+2 k, j-2} \bar{F}_{n+i, m}^{2 k, 2}$

The cases involving the sine and the cotangent are again very similar, and the relations among the coefficients in these cases are shown in Table 3 . The accuracy of the relations in the left-hand column of Table 3 is shown in Table 4. It was found that the recursive relations to compute the coefficients are all numerically accurate and stable, even for high degrees and orders and high index $j$, while larger numerical inaccuracies occur in the weighted summation over ALFs. Therefore, there is little difference with respect to numerical accuracy between the use of the different recursive relations in the right-hand column of Table 3 . The main differences are in the implementation, as was discussed above for the difference between Eqs. (22) and (24), and the efficiency. The required computation time for recursion from initial values of $j=2$, as in Eqs. (25) and (26), is approximately $60 \%$ of the recursion from the initial values of $j=1$, as in Eqs. (22) and (24), and is under a minute for computation of all coefficients up to degree and order 360 and index $j=32$ on a PC Pentium IV $2.4 \mathrm{GHz}$.

The absolute relative errors in the relations of Table 3 show little correlation with the degree and order of the ALF. There are, however, some irregular combinations of degree $n$ and order $m$ for which the absolute relative error is up to 4 or 5 orders larger than the averages shown in Table 4 , and this occurs in all relations tested, both in the unnormalised and the fully normalised form.

The major loss of accuracy occurs for high values of index $j$, especially in the case of the cotangent, as can be seen in Table 4. Furthermore, not surprisingly, there is also an increased loss of accuracy for certain values of $\theta$. The relation involving the sine is most accurate for $\theta$ close to $\frac{\pi}{2}(\bmod \pi)$, but becomes less accurate for $\theta$ close to $0(\bmod \pi)$. For the relation involving the cosine, naturally, the reverse pattern can be seen, while the relation involving the cotangent becomes inaccurate for both cases $\left(\theta\right.$ close to $\left.0\left(\bmod \frac{\pi}{2}\right)\right)$.

The unnormalised coefficients $E_{n}^{i j}$ show some properties that can facilitate their computation. First of all, they are independent of order $m$. Furthermore, from Table 1, it can be seen that

$E_{n}^{-1,1}=-E_{n}^{1,1}$

Due to the simplicity of the unnormalised coefficients $E_{n}^{i j}$, an analytic expression can be found that does not require any recurrence

$E_{n}^{i j}=\frac{(2 n+2 i+1)(2 n+i-j-1) ! !}{(-1)^{\frac{j-i}{2}}(2 n+i+j+1) ! !}\left(\begin{array}{c}j \\ \frac{i+j}{2}\end{array}\right)$

where !! is the double factorial operator. A proof for Eq. (34) is given in Appendix B. As can be seen in Table 4, this ana- 
lytic formula does not provide any advantages over the recursive approach with respect to accuracy and stability, but it is slightly more efficient. Analytic expressions for the fully normalised coefficients $\bar{E}_{n m}^{i j}$ and the other coefficients $F_{n m}^{i j}$ and $G_{n m}^{i j}$ similar to Eq. (34) may also exist, but will presumably be more complicated than Eq. (34) and provide little advantages over the recursive relations.

It is also useful in this respect to note that Eq. (34) allows computation of the coefficients in the product-sum formula of ALFs (Hwang 1995) without recursion if one of the ALFs has equal degree and order. Since

$P_{m m}=(2 m-1) ! ! \sin ^{m} \theta$

it follows that

$P_{m m} P_{r s}=(2 m-1) ! ! \sum_{j=0}^{m} E_{r}^{m-2 j, m} P_{m+r-2 j, m+s}$

The exact representation of the weights given in Eq. (34) can serve as an alternative to the recursive relation provided in Hwang (1995).

In a similar way to Eq. (27), two more expressions can be found for even $j$

$\cos ^{j} \theta P_{n m}=P_{n m}-\sum_{i=-j, 2}^{j} E_{n}^{i j} P_{n+i, m+j}, \quad j \in \mathbb{E}$

and

$\csc ^{j} \theta P_{n m}=\sum_{i=-j, 2}^{j} L_{n m}^{i j} P_{n, m+i}, \quad m \geq j, \quad j \in \mathbb{E}$

where csc denotes the cosecant (the multiplicative inverse of the sine) and the initial values are

$L_{n m}^{-2,2}=G_{n m}^{-2,2}, \quad L_{n m}^{0,2}=1+G_{n m}^{0,2}, \quad L_{m}^{2,2}=G_{m}^{2,2}$

Equations (37) and (38) will prove useful in the construction of identities among ALFs shown in section 5.

\section{Derivatives of ALFs}

In the case of first- or second-order derivatives of ALFs, similar types of expression can be found. These follow directly from well-known relations equating the derivatives of ALFs to a summation over ALFs and are all valid in both the unnormalised and the fully normalised case. The first-order derivative can be expressed as a summation over ALFs of equal degree $n$, or as a summation over ALFs of equal order $m$ (e.g. Abramowitz and Stegun 1972)

$$
\begin{aligned}
\frac{\partial P_{n m}}{\partial \theta} & =n \cot \theta P_{n m}-(n+m) \csc \theta P_{n-1, m} \\
& =-P_{n, m+1}+m \cot \theta P_{n m}
\end{aligned}
$$

Inserting the third equation in the left column of Table 1 into Eq. (41) yields

$$
\frac{\partial P_{n m}}{\partial \theta}=m G_{n m}^{-1,1} P_{n, m-1}+\left(m G_{m}^{1,1}-1\right) P_{n, m+1}
$$

Multiplying both sides of this equation by the sine, cosine or cotangent of angle $\theta$ gives, together with the equations in Table 1, expressions that equate the multiplication of one of these trigonometric functions with the derivative of an ALF to a weighted summation over ALFs, where the weights are independent of angle $\theta$. Only in the case of a cotangent, the weighted summation runs over ALFs of equal degree $n$, which is

$$
\begin{aligned}
\cot \theta \frac{\partial P_{n m}}{\partial \theta} & =m G_{n m}^{-2,2} P_{n, m-2}+\left(m G_{n m}^{0,2}-G_{n, m+1}^{-1,1}\right) P_{n m} \\
& +\left(m G_{n m}^{2,2}-G_{m+1}^{1,1}\right) P_{n, m+2}
\end{aligned}
$$

Equation (43) can be generalised using the third equation in the left column of Table 1

$\cot ^{j} \theta \frac{\partial P_{n m}}{\partial \theta}=\sum_{i=-j-1,2}^{j+1} M_{n m}^{i j} P_{n, m+i}$

where the coefficients $M_{n m}^{i j}$ can be computed using the same recursive relations as used to compute $G_{n m}^{i j}$ from the initial values given in Eq. (43).

From Eq. (40), a formula can be found for multiplication of the first derivative of ALFs by the sine of angle $\theta$ written as a summation over two ALFs of equal order $m$.

$$
\begin{aligned}
\sin \theta \frac{\partial P_{n m}}{\partial \theta} & =n \cos \theta P_{n m}-(n+m) P_{n-1, m} \\
& =\left(n F_{n m}^{-1,1}-n-m\right) P_{n-1, m}+n F_{n, m}^{1,1} P_{n+1, m}
\end{aligned}
$$

Equation (45) can easily be extended in a similar fashion to Eq. (17) yielding

$\sin \theta \cos ^{j} \theta \frac{\partial P_{n m}}{\partial \theta}=\sum_{i=-j-1,2}^{j+1} N_{n m}^{i j} P_{n+i, m}$

where the coefficients $N_{n m}^{i j}$ can be computed recursively using the same principles as those used to compute $F_{n m}^{i j}$. The case $j=1$ is, for example, often used in computation of ellipsoidal corrections in geoid computation (e.g. Moritz 1980).

The second-order derivatives of ALFs follow from the characteristic differential equation (e.g. Hobson 1931)

$$
\frac{\partial^{2} P_{n m}}{\partial \theta^{2}}=-\cot \theta \frac{\partial P_{n m}}{\partial \theta}-\left[n(n+1)-m^{2} \csc ^{2} \theta\right] P_{n m}
$$

Using Eqs. (38) and (43), Eq. (47) can be written as

$$
\begin{aligned}
\frac{\partial^{2} P_{n m}}{\partial \theta^{2}} & =m(m-1) G_{n m}^{-2,2} P_{n, m-2} \\
& +\left[m(m-1) G_{n m}^{0,2}+G_{n, m+1}^{-1,1}-n^{2}+m^{2}-n\right] P_{n m} \\
& +\left[m(m-1) G_{m}^{2,2}+G_{m+1}^{1,1}\right] P_{n, m+2}
\end{aligned}
$$

Thus, also second-order derivatives of ALFs, and - as such their products with powers of sine, cosine or cotangent, can be written as a weighted summation over ALFs, with weights only dependent on degree $n$ and order $m$. 


\section{Identities among ALFs}

Many of the formulae derived in the previous sections can be combined to provide identities among ALFs that involve no other dependency on angle $\theta$ than that contained in the ALFs themselves. There are many ways to establish such identities.

Firstly, Eq. (27) is equal to the first equation in the left column of Table 3 for even $j$. This yields relations among a minimum of six ALFs

$$
\sum_{i=-j, 2}^{j} K_{n m}^{i j} P_{n+i, m}=\sum_{i=-j, 2}^{j} E_{n}^{i j} P_{n+i, m+j}, \quad j \in \mathbb{E}
$$

The same concept can be applied to Eq. (37) and the second equation in the left column of Table 3, yielding exactly the same equation.

A second way to find an identity is to subsequently apply Eqs. (27) and (38). By applying these in two different orders, two identities can be obtained

$$
\begin{aligned}
P_{n m} & =\sin ^{j} \theta \csc ^{j} \theta P_{n m} \\
& =\sum_{i=-j, 2}^{j} \sum_{k=-j, 2}^{j} K_{n m}^{i j} L_{n+i, m}^{k j} P_{n+i, m+k} \\
& =\sum_{i=-j, 2}^{j} \sum_{k=-j, 2}^{j} K_{n, m+k}^{i j} L_{n m}^{k j} P_{n+i, m+k}, \\
& m \geq j, \quad j \in \mathbb{E}
\end{aligned}
$$

Eqs. (50) and (51) relate a minimum of nine ALFs, and are linearly independent.

A third way is to combine all three equations in the left column of Table 3

$\cos ^{j} \theta P_{n m}=\cot ^{j} \theta \sin ^{j} \theta P_{n m}, \quad j \in \mathbb{N}$

Equation (52), contrary to Eqs. (49) and (51), also holds for $j=1$, and it thus provides relations among a minimum of four ALFs. The cotangent and sine on the right-hand-side of Eq. (52) can be reversed, which means two different relations can again be found. These two relations are, however, linearly dependent.

$$
\begin{array}{r}
\sum_{i=-j, 2}^{j} F_{n m}^{i j} P_{n+i, m}=\sum_{i=-j, 2}^{j} \sum_{k=-j, 2}^{j} E_{n}^{i j} G_{n m}^{k j} P_{n+i, m+j+k} \\
=\sum_{i=-j, 2}^{j} \sum_{k=-j, 2}^{j} E_{n}^{i j} G_{n+i, m+j}^{k j} P_{n+i, m+j+k}
\end{array}
$$

For the case $j=1$ in unnormalised form, they degenerate to a simple equation

$$
\begin{aligned}
& (n+m)(n+m+1) P_{n-1, m}+P_{n-1, m+2} \\
& \quad=(n-m)(n-m+1) P_{n+1, m}+P_{n+1, m+2}
\end{aligned}
$$

but all identities derived above can, naturally, also be used in the fully normalised form. Finally, the equations for the derivatives can also be utilised. When Eq. (42) is multiplied by the sine of angle $\theta$ and the first equation in the left column of Table 1 is subsequently applied, it can be directly compared to Eq. (45). This also yields Eq. (54).

\section{Example of application: analytic computation of geopotential coefficients}

Analytic methods to compute geopotential coefficients from gravity anomalies on the ellipsoid form one example where the introduction of some of the above derived relations can prove useful. Most of these methods, such as the ones derived by Cruz (1986), Petrovskaya et al. (2001) and Sjöberg (2003), rely on an approximation of the boundary condition to the order of the first eccentricity of the reference ellipsoid $e^{2}$. At this level of approximation, Eq. (15) can be utilised to write the spherical harmonic coefficients of the Earth's disturbing potential $T_{n m}$ as a linear combination of coefficients that were computed in spherical approximation $T_{n m}^{0}$. In all three above-mentioned articles, the computation of $T_{n m}$ takes the form

$T_{n m}=\alpha_{n m} T_{n-2, m}^{0}+\beta_{n m} T_{n m}^{0}+\gamma_{n m} T_{n+2, m}^{0}$

where $\alpha_{n m}, \beta_{n m}$ and $\gamma_{n m}$ only depend on degree $n$, order $m$ and the definition of the reference ellipsoid and its normal gravity field. Sjöberg (2003) correctly notes that this approximation is not sufficient for higher degrees of harmonics. Cruz (1986) also acknowledges this, and attempts to solve the inaccuracies by including terms of order $e^{4}$ and $e^{6}$. In order to do so, he derives explicit relations that are equal to the cases $j=4$ and $j=6$ of Eq. (27). However, Gleason (1988) has shown that Cruz's solution nevertheless has severe shortcomings, even below degree 360.

Using Eq. (17) or Eq. (27), it is no longer necessary to apply any approximation to the fundamental equation of physical geodesy (Heiskanen and Moritz 1967, Eq. 2-148), which forms the boundary condition in this problem. Claessens and Featherstone (2005) have shown that the computation of spherical harmonic coefficients of the disturbing potential $T_{n m}$ can be expressed in a theoretically exact way as a weighted summation over the spherically approximated coefficients $T_{n m}^{0}$

$T_{n m}=\sum_{i=-\infty}^{\infty} \alpha_{n m i} T_{n+2 i, m}^{0}$

using Eq. (27). It is also shown in Claessens and Featherstone (2005) that the infinite series in Eq. (56) will converge for every degree $n$ and order $m$, and that the convergence rate is sufficiently fast for practical application, at least until degree and order 2160. Therefore, computation of geopotential coefficients as a summation over spherically approximated coefficients has now become feasible with very high numerical accuracy.

The analytic computation of geopotential coefficients is only one example where the new relations derived here can be applied in practice to generalise existing methods and thereby improve their accuracy. In similar fashion, Eq. (17) or Eq. (27) can be used to generalise the ellipsoidal corrections to Stokes's function and to geoid heights, providing exact relations rather than approximations, extending on the work by Moritz (1980), Martinec (1998), Heck and Seitz (2003) and Sjöberg (2003, 2004). 


\section{Conclusions}

Several general relations among both unnormalised and fully normalised associated Legendre functions (ALFs) have been derived, which can be used in various applications such as, for example, ellipsoidal corrections to geoid heights and computation of geopotential coefficients. These relations equate the product of an ALF with trigonometric functions to a weighted summation over ALFs. The weights only depend on the degree and order of the ALFs and can be computed recursively. Several recursive relations to compute the weights (shown in Table 3) were derived, and identities connecting the weight functions which facilitate their computation (Eqs. 30 and 34) were proven to hold. It is shown that the relations can also be applied in the case of first- and second-order derivatives of ALFs (Eqs. 42 and 48), and by combination of the aforementioned relations, new identities among ALFs were derived (Eqs. 49, 50, 51 and 53).

\section{Appendix A}

A proof for the identity given in Eq. (30) can be found by mathematical induction, where Eq. (30) forms the proposition

$\bar{F}_{n m}^{i j}=\bar{F}_{n+i, m}^{-i, j}$

It can easily be seen from Eqs. (9) and (10) that this proposition holds for the base case $i=j=1$. Assuming that the inductive hypothesis

$\bar{F}_{n m}^{p q}=\bar{F}_{n+p, m}^{-p, q}$

is true for all $p$ and $q$, the two propositions of the inductive step remain to be proven, i.e.

$\bar{F}_{n m}^{p+1, q}=\bar{F}_{n+p+1, m}^{-p-1, q}$

$\bar{F}_{n m}^{p, q+1}=\bar{F}_{n+p, m}^{-p, q+1}$

Inserting Eq. (24) in the left-hand-side of Eq. (59), and subsequently applying the inductive hypothesis in Eq. (58), gives an equation, which - according to Eq. (22) - is exactly the same as the right-hand side of Eq. (59)

$$
\begin{aligned}
\bar{F}_{n m}^{p+1, q} & =\bar{F}_{n m}^{p+2, q-1} \bar{F}_{n+p+2, m}^{-1,1}+\bar{F}_{n m}^{p, q-1} \bar{F}_{n+p, m}^{1,1} \\
& =\bar{F}_{n+p+2, m}^{-p-2, q-1} \bar{F}_{n+p+1, m}^{1,1}+\bar{F}_{n+p, m}^{-p, q-1} \bar{F}_{n+p+1, m}^{-1,1} \\
& =\bar{F}_{n+p+1, m}^{-p-1, q} \quad \text { Q.E.D. }
\end{aligned}
$$

A similar procedure can be followed to prove Eq. (60)

$$
\begin{aligned}
\bar{F}_{n m}^{p, q+1} & =\bar{F}_{n m}^{-1,1} \bar{F}_{n-1, m}^{p+1, q}+\bar{F}_{n m}^{1,1} \bar{F}_{n+1, m}^{p-1, q} \\
& =\bar{F}_{n-1, m}^{1,1} \bar{F}_{n+p, m}^{-p-1, q}+\bar{F}_{n+1, m}^{-1,1} \bar{F}_{n+p, m}^{-p+1, q} \\
& =\bar{F}_{n+p, m}^{-p, q+1} \quad \text { Q.E.D. }
\end{aligned}
$$

Since the base case and the inductive step are true, the proposition in Eq. (57) is true for all $i$ and $j$.

\section{Appendix B}

A proof for the identity given in Eq. (34) can be found by mathematical induction, where Eq. (34) forms the proposition

$E_{n}^{i j}=\frac{(2 n+2 i+1)(2 n+i-j-1) ! !}{(-1)^{\frac{j-i}{2}}(2 n+i+j+1) ! !}\left(\begin{array}{c}j \\ \frac{i+j}{2}\end{array}\right)$

It can be seen from Table 1 that this proposition holds for the base cases $i=j=1$ and $i=-1, j=1$. Assuming that the inductive hypothesis

$E_{n}^{p q}=\frac{(2 n+2 p+1)(2 n+p-q-1) ! !}{(-1)^{\frac{q-p}{2}}(2 n+p+q+1) ! !}\left(\begin{array}{c}q \\ \frac{p+q}{2}\end{array}\right)$

is true for all $p$ and $q$, the two propositions of the inductive step remain to be proven, i.e.

$E_{n}^{p+1, q}=\frac{(2 n+2 p+3)(2 n+p-q) ! !}{(-1)^{\frac{q-p-1}{2}}(2 n+p+q+2) ! !}\left(\begin{array}{c}q \\ \frac{p+q+1}{2}\end{array}\right)$
$E_{n}^{p, q+1}=\frac{(2 n+2 p+1)(2 n+p-q-2) ! !}{(-1)^{\frac{q-p+1}{2}}(2 n+p+q) ! !}\left(\begin{array}{c}q \\ \frac{p+q+1}{2}\end{array}\right)$

Inserting Eq. (64) into the second equation for $E_{n}^{i j}$ in Table 3 and making use of Eq. (33) gives

$$
\begin{aligned}
E_{n}^{p+1, q} & =E_{n}^{1,1}\left(E_{n+1}^{p, q-1}-E_{n-1}^{p+2, q-1}\right) \\
& =\frac{2 n+2 p+3}{2 n+1}(-1)^{\frac{q-p-1}{2}} \frac{(2 n+p-q) ! !}{(2 n+p+q+2) ! !} \\
& \times\left[(2 n+p-q+2)\left(\begin{array}{c}
q-1 \\
\frac{p+q-1}{2}
\end{array}\right)\right. \\
& \left.+(2 n+p+q+2)\left(\begin{array}{c}
q-1 \\
\frac{p+q+1}{2}
\end{array}\right)\right]
\end{aligned}
$$

The latter part between the square brackets can be simplified using well-known identities among binomial coefficients

$$
\left(\begin{array}{l}
p \\
q
\end{array}\right)=\left(\begin{array}{l}
p-1 \\
q-1
\end{array}\right)+\left(\begin{array}{c}
p-1 \\
q
\end{array}\right) \quad(\text { Pascal's formula })
$$

and

$$
\left(\begin{array}{l}
p \\
q
\end{array}\right)=\frac{p}{q}\left(\begin{array}{l}
p-1 \\
q-1
\end{array}\right)=\frac{p}{p-q}\left(\begin{array}{c}
p-1 \\
q
\end{array}\right)
$$

which concludes the proof

$$
\begin{aligned}
E_{n}^{p+1, q} & =\frac{2 n+2 p+3}{2 n+1}(-1)^{\frac{q-p-1}{2}} \frac{(2 n+p-q) ! !}{(2 n+p+q+2) ! !} \\
& \times\left[(2 n+1)\left\{\left(\begin{array}{c}
q-1 \\
\frac{p+q-1}{2}
\end{array}\right)+\left(\begin{array}{c}
q-1 \\
\frac{p+q+1}{2}
\end{array}\right)\right\}\right. \\
& \left.+(p-q+1)\left(\begin{array}{c}
q-1 \\
\frac{p+q-1}{2}
\end{array}\right)+(p+q+1)\left(\begin{array}{c}
q-1 \\
\frac{p+q+1}{2}
\end{array}\right)\right] \\
& =\frac{2 n+2 p+3}{2 n+1}(-1)^{\frac{q-p-1}{2}} \frac{(2 n+p-q) ! !}{(2 n+p+q+2) ! !} \\
& \times(2 n+1)\left(\begin{array}{c}
q \\
\frac{p+q+1}{2}
\end{array}\right) \quad \text { Q.E.D. }
\end{aligned}
$$

A similar procedure can be followed to prove Eq. (66). Then, since the base cases and the inductive step are true, the proposition in Eq. (63) is true for all $i$ and $j$. 


\section{References}

Abramowitz M, Stegun IA (1972) Handbook of mathematical functions, 9th edn. Dover Publications, New York

Blatt JM, Weisskopf VF (1962) Theoretical Nuclear Physics. John Wiley \& Sons, New York

Claessens SJ, Featherstone WE (2005) Computation of geopotential coefficients from gravity anomalies on the ellipsoid. In: Sanso F. (Ed.) "Proceedings of the International Association of Geodesy: A Window on the Future of Geodesy". Springer, IAG Symposia Series 128: 459-464

Cruz JY (1986) Ellipsoidal correction to potential coefficients obtained from gravity anomaly data on the ellipsoid. Rep 371, Department of Geodetic Science and Surveying, The Ohio State University, Columbus

Gerstl M (1980) On the recursive computation of the integrals of the associated Legendre functions. Manuscr Geod 5: 181-199

Giacaglia GED (1980) Transformations of spherical harmonics and applications to geodesy and satellite theory. Stud Geophys et Geod 24: 1-11

Gleason DM (1988) Comparing ellipsoidal corrections to the transformation between the geopotential's spherical and ellipsoidal spectrum. Manuscr Geod 13: 114-129

Heck B, Seitz K (2003) Solutions of the linearized geodetic boundary value problem for an ellipsoidal boundary to order $e^{3}$. J Geod 77: $182-192$

Heiskanen WA, Moritz H (1967) Physical Geodesy. W.H. Freeman and Co., San Francisco

Hobson EW (1931) The theory of spherical and ellipsoidal harmonics. Cambridge University Press, Cambridge

Holmes SA, Featherstone WE (2002) A unified approach to the Clenshaw summation and the recursive computation of very high degree and order normalised associated Legendre functions. J Geod 76: 279-299

Hwang C (1995) A method for computing the coefficients in the product-sum formula of associated Legendre functions. J Geod 70: $110-116$

Koop R, Stelpstra D (1989) On the computation of the gravitational potential and its first and second order derivatives. Manuscr Geod 14: $373-382$

Lemoine FG, Kenyon SC, Factor JK, Trimmer RG, Pavlis NK, Chinn DS, Cox CM, Klosko SM, Luthcke SB, Torrence MH, Wang YM, Williamson RG, Pavlis EC, Rapp RH, Olson TR (1998) The development of the joint NASA GSFC and the National Imagery and Mapping Agency (NIMA) Geopotential Model EGM96. NASA/TP-1998-206861, Goddard Space Flight Center, Greenbelt

MacRobert TM (1967) Spherical Harmonics, 3rd edn. Pergamon Press Ltd, Oxford

Martinec Z (1998) Construction of Green's function for the Stokes boundary-value problem with ellipsoidal corrections in the boundary condition. J Geod 72: 460-472

Moritz H (1980) Advanced physical geodesy. Wichmann Verlag, Karlsruhe

Percival WJ, Burkey D, Heavens A, Taylor A, Cole S, Peacock JA, Baugh CM, Bland-Hawthorn J, Bridges T, Cannon R, Colless M, Collins C, Couch W, Dalton G, De Propis R, Driver SP, Efstathiou G, Ellis RS, Frenk CS, Glazebrook K, Jackson C, Lahav O, Lewis I, Lumsden S, Maddox S, Norberg P, Peterson BA, Sutherland W, Taylor K (2004) The 2dF Galaxy Redshift Survey: spherical harmonics analysis of fluctuations in the final catalogue. Mon Not R Astron Soc 353: 1201-1218.

Petrovskaya MS, Vershkov AN, Pavlis NK (2001) New analytical and numerical approaches for geopotential modeling. J Geod 75: 661-672

Reigber C, Balmino G, Schwintzer P, Biancale R, Bode A, Lemoine JM, König R, Loyer S, Neumayer H, Marty JC, Barthelmes F, Perosanz F, Zhu SY (2002) A high-quality global gravity field model from CHAMP GPS tracking data and accelerometry (EIGEN-1S). Geophys Res Lett 29:37
Rothwell EJ, Cloud MJ (2001) Electromagnetics. CRC Press, Boca Raton

Sjöberg LE (2003) Ellipsoidal corrections to order $e^{2}$ of geopotential coefficients and Stokes's formula. J Geod 77: 139-149

Sjöberg LE (2004) A spherical harmonic representation of the ellipsoidal correction to the modified Stokes formula. J Geod 78: $180-186$ 